\title{
The role of autophagy in tumour development and cancer therapy
}

\section{Mathias T. Rosenfeldt and Kevin M. Ryan*}

Autophagy is a catabolic membrane-trafficking process that leads to sequestration and degradation of intracellular material within lysosomes. It is executed at basal levels in every cell and promotes cellular homeostasis by regulating organelle and protein turnover. In response to various forms of cellular stress, however, the levels and cargoes of autophagy can be modulated. In nutrient-deprived states, for example, autophagy can be activated to degrade cargoes for cell-autonomous energy production to promote cell survival. In other contexts, in contrast, autophagy has been shown to contribute to cell death. Given these dual effects in regulating cell viability, it is no surprise that autophagy has implications in both the genesis and treatment of malignant disease. In this review, we provide a comprehensive appraisal of the way in which oncogenes and tumour suppressor genes regulate autophagy. In addition, we address the current evidence from human cancer and animal models that has aided our understanding of the role of autophagy in tumour progression. Finally, the potential for targeting autophagy therapeutically is discussed in light of the functions of autophagy at different stages of tumour progression and in normal tissues.

During the genesis of cancer, tumour cells experience various forms of intracellular and extracellular stress. This hostile situation results in damage to cellular proteins, the production of reactive oxygen species (ROS) and the replication of cells with heritable DNA damage. Tumour cells must therefore utilise homeostatic mechanisms in order to maintain sufficient energy and integrity in order to survive. Macroautophagy (hereafter referred to autophagy for simplicity) is one process within the cell that can serve to support these demands, through the lysosomal-mediated degradation of cellular proteins and organelles. This not only results in the removal of damaged cellular constituents, but also, where required, can provide the catabolic intermediates for intracellular production of ATP when exogenous energy supplies are limited (Refs 1,2,3).

As outlined above, it would seem that autophagy, as a result of its prosurvival function, could serve as a potent oncogenic mechanism to promote tumour cell survival.

Tumour Cell Death Laboratory, Beatson Institute for Cancer Research, Glasgow, G61 1BD, UK.

*Corresponding author: Kevin M. Ryan, Beatson Institute for Cancer Research, Garscube Estate, Switchback Road, Glasgow, G61 1BD, UK. Tel: +44 1413 303655; Fax: +44 1419 426521; E-mail:

k.ryan@beatson.gla.ac.uk

Accession information: doi:10.1017/S1462399409001306; Vol. 11; e36; December 2009 (c) Cambridge University Press 2009. Re-use permitted under a Creative Commons Licence - by-nc-sa. 
Paradoxically, however, although a clear role in cell survival undoubtedly exists, evidence also points to a tumour-suppressive role: downregulation of several autophagy genes occurs in human cancer, mouse models where critical autophagy regulators have been deleted support a role for autophagy in tumour suppression, and autophagy has been implicated in cell death and oncogene-induced senescence (Refs 4, 5, 6, 7). A conundrum currently exists, therefore, as to the role of autophagy in cancer, with perhaps different roles being played at different stages and in different tumour types (Ref. 8). Understanding this issue in great detail is seemingly critical if we are to consider targeting autophagy - either positively or negatively for therapeutic gain.

\section{Mechanisms of autophagy}

Autophagy when translated from the Greek literally means 'self-eating', and comprises a multistep process of sequestration and subsequent degradation of intracellular material within specialised compartments (Refs 9, 10). Autophagy is orchestrated by a subset of genes that were originally identified in yeast and are called autophagy-related genes (ATG), many of which have mammalian orthologues (Refs 10, 11). Autophagy can be divided into different stages that ultimately result in lysosomal breakdown of cytoplasmatic material: initiation; autophagosome formation (nucleation, elongation and completion); and maturation and degradation. These are discussed in detail below and depicted in Figure 1.

\section{Initiation}

Initiation in mammalian cells starts with the activation of a serine/threonine kinase complex that contains ULK1/2 (orthologues of the yeast protein Atg1), ATG13 and FIP200 (RB1CC1) This complex transfers signals from the nutrient-sensing mTOR kinase (MTOR; mammalian/mechanistic target of rapamycin) to initiate autophagy (Refs 12, 13, 14, 15). Inhibition of mTOR-induced phosphorylation of ULK and ATG13 liberates the kinase activity of ULK, which subsequently phosphorylates itself, ATG13 and FIP200. The ULK complex then accumulates at the initiating focus of vesicle formation: the isolation membrane or phagophore (Refs 13, 14, 15).

\section{Autophagosome formation}

Vesicle nucleation - the further development of the isolation membrane/phagophore - critically depends on the activity of the class III phosphoinositide 3-kinase (PI3K-III) hVps34 (PIK3C3; the orthologue of yeast Vps34) and its formation of a complex with Beclin 1 (yeast Atg6) and p150/hVps35 (PIK3R4; yeast Vps15) (Refs 16, 17).

Vesicle elongation and completion from the isolation membrane/phagophore to a nascent autophagosome and the completed, closed autophagosome is mediated by two ubiquitinlike conjugation systems: the ATG12 and ATG8 conjugation systems (Ref. 18). The ubiquitin-like ATG12 is activated by ATG7 and then temporarily binds to the E2-like enzyme ATG10 before being transferred to ATG5. ATG5 further reacts with ATG16 to form a multimeric complex of ATG12-ATG5-ATG16 (Refs 19, 20). In mammalian cells, several orthologues of yeast Atg8 have been identified: MAP1LC3 (LC3), GABARAPL2 (GATE16), GABARAP and GABARAPL1 (ATG8L). They are subjected to modification steps similar to those of their yeast counterpart. The processing of the autophagosome marker LC3 has been investigated most thoroughly, and its stepwise conversion from a cytosolic to a membranebound form is exploited for the experimental measurement of autophagy (Ref. 21). LC3 is synthesised as a precursor protein proLC3 and is immediately processed to LC3-I by ATG4 through cleavage of the C-terminal amino acid. LC3 maturation completes with the reversible conjugation of LC3-I to phosphatidylethanolamine (PE) at the C-terminus by ATG3 and ATG7 to form LC3-II on the surface of autophagosomes (Refs 19, 20).

Both the ATG12-ATG5-ATG16 complex and LC3-II are essential for autophagy. The ATG12-ATG5-ATG16 complex is required for targeting of LC3 to the autophagosomal membrane and accelerated conjugation of LC3 to PE. Atg8, the yeast counterpart of LC3, controls the expansion of the phagophore and the amount of Atg8 directly correlates to the size of the autophagosomes (Refs 19, 22). The different stages of autophagosome formation are intertwined and difficult to separate. Therefore the assignment of the different ATGs to corresponding stages and autophagic vesicles is to some degree academic.

Accession information: doi:10.1017/S1462399409001306; Vol. 11; e36; December 2009 (c) Cambridge University Press 2009. Re-use permitted under a Creative Commons Licence - by-nc-sa. 
Figure 1. Mechanisms of autophagy. Following an initiating event, ATGs orchestrate the formation of autophagic vesicles from the phagophore/isolation membrane to the autophagosome and finally the autolysosome. The ULK-ATG13-FIP200 and the Beclin-1-hVps34-p150 complexes mediate early nucleation events, whereas the two ubiquitin-like conjugation systems (ATG5-ATG12 and LC3-II) direct vesicle elongation and autophagosome formation. Cellular material is finally sequestered within the autophagosome and thereby separated from the cytoplasm. Intracellular material is degraded in autolysosomes, which result from a fusion of lysosomes with autophagosomes. Importantly, autophagy is the complete process from initiation to degradation and not just the accumulation of autophagosomes. Abbreviations: ATG, autophagy-related protein; Beclin 1, coiled-coil myosin-like Bcl-2-interacting protein; FIP200, 200 kDa FAK family kinase-interacting protein; hVps34, human vacuolar protein sorting-associated protein 34; LC3, (microtubule-associated protein) light chain 3; mTOR, mammalian/mechanistic target of rapamycin; p150, regulatory subunit of hVps34; P, phosphorylation; ULK1/2, unc-51-like kinase 1/2.

Accession information: doi:10.1017/S1462399409001306; Vol. 11; e36; December 2009 (c) Cambridge University Press 2009. Re-use permitted under a Creative Commons Licence - by-nc-sa. 


\section{Maturation and degradation}

The maturation process encompasses the fusion of autophagosomes with lysosomes to form the end-stage vesicle of autophagy: the autolysosome. Molecular mechanisms of autophagosome maturation are only recently emerging and involve the actions of lysosomal proteins LAMP1 and LAMP2, the small GTPase Rab7 (RAB7A), UVRAG (the protein product of the ultraviolet-radiation-resistance-associated gene) and others (Ref. 9). The tumour suppressor UVRAG not only regulates the interaction of Beclin 1 and $\mathrm{hVps} 34$ at the stage of vesicle nucleation but also plays an important role in the maturation step. UVRAG directs so-called tethering proteins (i.e. proteins that connect the autophagosome to its target) to the autophagosomal membrane and thereby activates Rab7 to facilitate fusion with lysosomes (Ref. 17).

The final autolysosome is an acidic vesicle wherein the intracellular material gets degraded by lysosomal hydrolases, especially cathepsins. Amino acids and other constituent parts generated in this catabolic process are then released from the autolysosome to fuel cellular resources. In yeast, amino acid efflux is mediated in part via Atg22 (Ref. 23). Although mammalian lysosomal amino acid transporters have been described, a clear orthologue of Atg22 in these higher species is yet to be defined.

\section{Autophagy and cancer - from human mutations to mouse models}

Malignant transformation of a normal cell into a cancer cell results from accumulation of several mutations that override cellular safeguard mechanisms such as apoptosis or oncogeneinduced senescence (Ref. 24). Currently it is unclear how autophagy is to be interpreted in the context of cancer. As outlined above, the unsolved paradox is that on the one hand its cytoprotective traits could clearly promote survival of both cancer and normal cells (Refs 1 , 3 ), whereas on the other hand autophagy might suppress tumour growth through its connection to cell death, senescence and oxidative stress (Refs 6, 7, 25, 26, 27, 28).

The genetic evidence that autophagy and cancer are linked is overwhelming (Refs 11, 29, 30). We detail here in a piecemeal fashion the data that have accumulated from humans and mouse models about the function of ATGs, their direct binding partners and their roles in either protecting against or promoting cancer.

\section{Beclin 1}

Beclin 1 (BECN1) is a critical autophagyregulating gene and provided the first human genetic link between autophagy and cancer. It is monoallelically deleted in $\sim 50 \%$ of human breast, ovarian and prostate cancers and expressed only at low levels in brain tumours (Refs 31, 32, 33). Subsequent studies of genetically modified mice revealed that while bi-allelic loss of Becn1 is embryonically lethal, mice that retain one copy of the gene are viable, but have a higher incidence of lymphomas, lung and liver carcinomas, as well as mammary hyperplasia and accelerated formation of premalignant lesions induced by hepatitis B virus, compared with wild-type animals (Refs 34, 35). In addition, reintroduction of $B E C N 1$ into human MCF7 breast carcinoma cells, which express only trace amounts of Beclin 1, restores their autophagic capacity and inhibits their tumour-forming potential (Ref. 32). Beclin 1 is therefore a haploinsufficient tumour suppressor (Refs 34, 35).

\section{ATG5 and ATG7}

ATG5 and ATG7 are two other critical autophagy regulators that have been deleted in experimental animal models. Unlike complete deletion of Becn1, deficiency in ATG5 or ATG7 is not embryonically lethal and mice are born normal without any apparent abnormalities; however, both $A \operatorname{tg} 5^{-1-}$ and $A \operatorname{tg} 7^{-1-}$ mice die within $24 \mathrm{~h}$ after birth, presumably as a result of an inability to compensate the neonatal starvation period that occurs after being cut off from the maternal circulation, when autophagy is normally transiently upregulated (Refs 3,36 ). Mice hemizygous for either Atg5 or Atg7 are born viable and develop normally. In contrast to Becn1-hemizygous animals, however, they do not develop tumours (Refs 3, 36). Intercrossing tumour-prone mice to recently developed conditional knockout mice for Atg5 and Atg7 (Refs 36, 37) will seemingly be required for the spatial and temporal dissection of the contribution of these genes to cancer development.

\section{ATG4}

Yeast Atg4 has four mammalian orthologues, of which ATG4C (autophagin-3) is most widely

Accession information: doi:10.1017/S1462399409001306; Vol. 11; e36; December 2009 (c) Cambridge University Press 2009. Re-use permitted under a Creative Commons Licence - by-nc-sa. 
expressed. ATG4C-knockout animals are born viable, survive the neonatal starvation period and develop normally (Ref. 38). Upon nutrient restriction they show organ-specific reduction of autophagy in the diaphragm. Autophagic activity at basal levels is normal. Spontaneous tumours arise at the same frequency as in wildtype controls; however, they do have a higher susceptibility to develop fibrosarcomas after chemical carcinogenesis (Ref. 38). A functional redundancy of the different ATG4 proteins in mammals might explain the notion that ATG4C deficiency does not alter basal autophagic activity in vivo and the fact that ATG4C-deficient mice survive the neonatal starvation period (Ref. 38).

\section{UVRAG}

UVRAG is a tumour suppressor gene that is monoallelically deleted in a significant proportion of human colorectal carcinomas, and UVRAG expression in human HCT116 colon carcinoma cells reduces the tumourigenic potential of these cells in xenograft studies (Ref. 16). Furthermore, UVRAG is a target for frameshift mutations in colorectal and gastric carcinomas with microsatellite instability (Ref. 39).

A role for autophagy deficiency in intestinal tumourigenesis is further supported by the fact that frameshift mutations in other autophagyrelated genes - ATG2B, ATG5, ATG9B and ATG12 - have been found in up to $28 \%$ of human gastric and colorectal cancers with microsatellite instability (Ref. 40). However the significance of this finding is yet to be determined.

\section{BIF1}

BIF1 (SH3GLB1) is another protein that positively regulates autophagy, through interaction with UVRAG and Beclin 1 (Ref. 41). B1F1-knockout mice are born viable and develop normally, without any apparent abnormalities except for an enlarged spleen. However they do have a higher propensity to develop spontaneous cancer (mainly lymphomas and to a lesser extent solid tumours) at an average age of 12 months. BIF1 is thus a positive regulator of autophagy and a potential tumour suppressor (Ref. 41).

\section{Summary}

In summary, the available data from humans and mouse models where ATGs or their direct positive modulators are lost or expressed at low levels point towards a tumour-suppressive role of autophagy. It is important to keep in mind, however, that in all the above mouse models autophagy inhibition involved deletion of the gene in the target cells embryonically. As a result, all the pathologies observed arose from cells that had always had impaired autophagy. These experiments thus do not clarify the role of acute autophagy loss at a later developmental stage, for instance in an established tumour, a scenario that likely occurs in vivo. In order to address this important question, further analysis is therefore required of conditional autophagy-deficient animals, by using inducible Cre recombinases so that gene deletion can be achieved in the adult animal in a tissue- and tumour-specific manner.

Genetic regulation of autophagy in cancer As indicated above, the control of autophagy is multifactorial and incompletely understood, but there is an emerging trend that tumour suppressors induce autophagy whereas oncogenes have the opposite effect (Refs 5, 42). Many oncogenic and tumour-suppressive effects impact ultimately on mTOR and complexes of Beclin 1 and hVps34 (Beclin-1hVps34), which are central to autophagy regulation in many contexts (Ref. 43). These complexes therefore merit prior discussion in order to fully understand the control of autophagy by classic oncogenes and tumour suppressors (Fig. 2).

\section{mTOR is a gateway for the control of autophagy}

The serine/threonine kinase mTOR is a focal point for the action of many oncogenic and metabolic events as well as the control of autophagy. Many regulators of mTOR signalling, especially the PI3K-AKT-PTEN pathway, are frequently deregulated in human tumours (Ref. 44). mTOR is part of two different protein complexes: mTORC1 and mTORC2 (Ref. 45). These have different components, most notably raptor (RAPTOR; regulatory-associated protein of MTOR, complex 1) for mTORC1 and rictor (RICTOR; rapamycin-insensitive companion of MTOR, complex 2) for mTORC2 (Refs 46, 47). Activation of mTORC1 leads to protein synthesis via the actions of S6K1

Accession information: doi:10.1017/S1462399409001306; Vol. 11; e36; December 2009 (c) Cambridge University Press 2009. Re-use permitted under a Creative Commons Licence - by-nc-sa. 
(RPS6KB1, ribosomal protein S6 kinase, $70 \mathrm{kDa}$, polypeptide 1) and 4EBP1 (EIF4EBP1, eukaryotic translation initiation factor $4 \mathrm{E}$ binding protein 1). By contrast, mTORC2 is less well understood. It activates AKT via phosphorylation and enhances the expression of HIF-1 $\alpha$ (HIF1A; hypoxia-inducible factor
$1 \alpha)$ (Refs 48, 49). While driving translation, mTOR also inhibits autophagy and this effect is mediated via mTORC1 and its interaction with the ULK-ATG13-FIP200 complex. The net effect of mTOR activation is cell growth, proliferation and cell survival (Refs 45, 50).

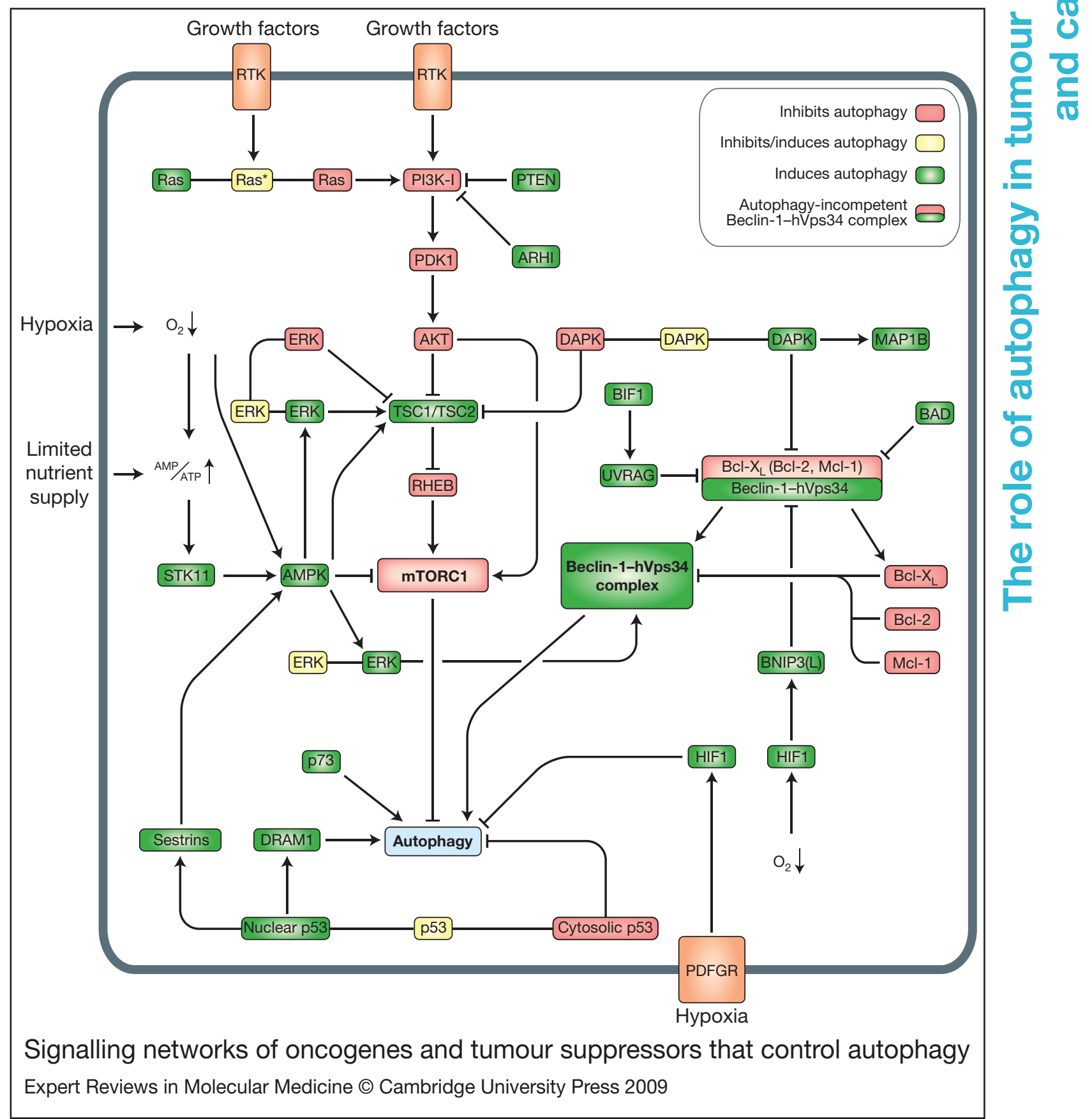

Figure 2. Signalling networks of oncogenes and tumour suppressors that control autophagy. (See next page for legend.)

Accession information: doi:10.1017/S1462399409001306; Vol. 11; e36; December 2009 (c) Cambridge University Press 2009. Re-use permitted under a Creative Commons Licence - by-nc-sa. 
Figure 2. Signalling networks of oncogenes and tumour suppressors that control autophagy. (See previous page for figure.) A multitude of pathways that are commonly deregulated in cancer control autophagy via its master switches mTOR and the complex of Beclin 1 and hVps34 (Beclin-1-hVps34). Hypoxia and limited nutrient supply are frequently encountered in tumours and lead to activation of autophagy via engagement of the same networks. Autophagy-inducing regulators are depicted in green, inhibiting regulators in red, and those that have a dual effect on autophagy in yellow. The asterisk indicates that the mechanism of Rasinduced upregulation of autophagy in senescence remains to be fully elucidated. Abbreviations: AKT, RACalpha serine/threonine-protein kinase; AMPK, AMP-activated protein kinase; ARHI, aplasia Ras homologue member I (also known as DIRAS3 for DIRAS family, GTP-binding RAS-like 3); BAD, Bcl-2-associated antagonist of cell death; Bcl-2, B-cell CLL/lymphoma 2 (apoptosis regulator); $\mathrm{BCl}-\mathrm{X}_{\mathrm{L}}$, B-cell lymphoma-extra large; Beclin 1, coiled-coil myosin-like Bcl-2-interacting protein; BIF1, endophilin B1; BNIP3(L), BCL2/ adenovirus E1B 19 kDa protein-interacting protein 3-(like); DAPK, death-associated protein kinase; DRAM1, damage-regulated autophagy modulator protein 1; ERK, extracellular-signal-regulated kinase; HIF1, hypoxia-inducible factor 1; MAP1B, microtubule-associated protein 1B; Mcl-1, induced myeloid leukaemia cell differentiation protein; mTORC1, mTOR complex 1; p53, cellular tumour antigen p53; p73, tumour protein p73; PDGFR, platelet-derived growth factors receptor; PDK1, 3-phosphoinositide-dependent protein kinase 1; PI3K-I, phosphoinositide 3-kinase class I; PTEN, phosphatase and tensin homologue; Ras, GTPase Ras; RTK, receptor tyrosine kinase; RHEB, Ras homologue enriched in brain; STK11, serine/ threonine-protein kinase 11 (also known as LKB1); TSC1, hamartin, tuberous sclerosis 1 protein; TSC2, tuberin, tuberous sclerosis 2 protein; UVRAG, UV-radiation-resistance-associated gene.

\section{The Beclin-1-hVps34 complex is a critical target for the regulation of autophagy} mTOR activity is pivotal in determining whether the net effect from signals within the cell is pro- or anti-autophagic. Another important regulator, downstream of mTOR, is the Beclin-1-hVps34 complex (Ref. 43). Beclin 1 was initially identified as a Bcl-2 (BCL2)-binding protein and was found to have tumour suppressor functions (Ref. 32). It has emerged, however, that Beclin 1 function is multifaceted and not restricted to the regulation of autophagy. It is part of a multiprotein complex that regulates autophagy through variation of its composition (Fig. 3). Beclin 1 forms a heterodimer that is stabilised via binding to antiapoptotic members of the Bcl-2 family [Bcl-2, Bcl- $X_{\mathrm{L}}$ (BCL2L1), Mcl-1 (MCL1)] through its Bcl-2-homology domain 3 (BH3) domain (Refs 51, 52). Monomerisation of Beclin 1 is required for it to associate with and activate the PI3K-III hVps34, which is critical for the formation of autophagosomes. Therefore, tumour-associated perturbations of antiapoptotic Bcl-2 family members can cause profound inhibition of autophagy by preventing the formation of a Beclin-1-hVps34 complex (Ref. 53).

The Beclin-1-hVps34-p150 core complex is complemented by different regulatory proteins that bind to Beclin 1 through domains other than $\mathrm{BH} 3$ and define its role in autophagy. To date, three different forms of the complex have been identified, all of which have different functions. They contain either ATG14/ BARKOR (Beclin-1-associated autophagyrelated key regulator) or UVRAG alone, or both UVRAG and Rubicon (RUN domain and cysteine-rich domain containing). It has been proposed that the ATG14/BARKORcontaining complex targets hVps34 activity towards the isolation membrane and thus functions in autophagosome formation, and that the UVRAG-containing complex promotes autophagosome and endosome maturation (Ref. 54). ATG14/BARKOR positively regulates autophagy, promotes translocation of Beclin 1 to autophagosomes and competes with UVRAG for the same binding site on Beclin 1 (Ref. 55). By contrast, the UVRAG- and Rubiconcontaining complex suppresses autophagosome and endosome maturation (Refs 56, 57). Knockdown of ATG14/BARKOR inhibits autophagy whereas knockdown of Rubicon has the opposite effect, providing further evidence that Beclin 1 has multiple roles in autophagy through the formation of complexes with opposing actions (Fig. 3). Although perturbation of Beclin 1 clearly has implications for cancer, a role for ATG14/BARKOR in cancer has not yet been defined. AMBRA1 is another protein that binds the Beclin-1-hVps34 complex, with a role in the regulation of autophagy in neuronal development (Ref. 58). However, as with ATG14/BARKOR, its role in autophagy regulation has also not yet been extended to cancer. 
Figure 3. Regulation of autophagy by Beclin 1 complexes. Beclin 1 exerts its function on autophagy only within a multimeric protein complex. It has diverse protein-binding domains that allow the formation of different complexes with opposing functions on autophagy. Certain stimuli, for example starvation, disrupt the binding of Bcl-2 proteins from the $\mathrm{BH} 3$ domain of Beclin 1. Beclin 1 can then bind and activate the phosphoinositide 3-kinase hVps34. Currently four regulators of the Beclin-1-hVps34 complex have been identified that determine its pro- or anti-autophagic activity. Autophagy-inducing regulators/complexes are depicted in green, inhibiting regulators/complexes in red, and those that have an undetermined role in yellow. Abbreviations: AMBRA1, activating molecule in Beclin-1-regulated autophagy protein 1; ATG, autophagy-related protein; BARKOR, Beclin-1-associated autophagy-related key regulator; Bcl-2, B-cell CLL/lymphoma 2; Bcl-X L, B-cell lymphoma-extra large; Beclin 1, coiled-coil myosin-like Bcl-2-interacting protein; $\mathrm{BH} 3, \mathrm{Bcl}-2$ homology domain 3; ECD, evolutionarily conserved domain; Mcl-1, induced myeloid leukaemia cell differentiation protein; Rubicon, RUN domain and cysteine-rich domain containing, Beclin-1interacting protein; UVRAG, ultraviolet-radiation-resistance-associated gene.

\section{Oncogenes and tumour suppressors}

As outlined above, many oncogenes and tumour suppressors exert opposing effects on autophagy, often through direct or indirect regulation of mTOR and Beclin-1-hVps34, but also through currently unknown mechanisms. In the following examples we outline the reported effects of tumour-associated proteins on both autophagy and apoptosis.

\section{PI3K-I}

PI3K-I (class I phosphoinositide 3-kinase) is a lipid kinase activated via receptor tyrosine kinases (RTKs) by multiple mechanisms (Refs 44,59). This leads to cell growth and cell proliferation and the concomitant inhibition of autophagy by activation of mTOR. Since RTKs and their downstream effectors are frequently mutated in cancer, activation in the PI3K-I pathway is very common in sporadic tumours (Ref. 59). Activated PI3K-I generates the second messenger phosphatidylinositol 3,4,5trisphosphate $\left[\operatorname{Ptd} \operatorname{Ins}(3,4,5) P_{3}\right]$, which then leads to activation/phosphorylation of AKT via 3-phosphoinositide-dependent protein kinase 1 (PDPK1/PDK1). AKT phosphorylates and

Accession information: doi:10.1017/S1462399409001306; Vol. 11; e36; December 2009 (c) Cambridge University Press 2009. Re-use permitted under a Creative Commons Licence - by-nc-sa. 
inhibits tuberous sclerosis protein 2 (TSC2), which as a result permits RheB (RHEB) to activate mTORC1 (Ref. 45). AKT can also activate mTOR independently of TSC2, via PRAS40 (AKT1S1; proline-rich AKT-substrate $40 \mathrm{kDa}$ ). In summary, the net effect of PI3K-I activation is activation of mTOR with subsequent inhibition of autophagy (Refs 45,50$)$.

\section{PTEN}

PTEN (phosphatase with tensin homologue) is mutated in multiple cancer types and is the major target for mutation in Cowden disease, a cancer predisposition syndrome (Ref. 60). PTEN negatively regulates PI3K-I and thereby alleviates the inhibitory effects of mTOR on autophagy. Consequently, inactivation of PTEN suppresses autophagy. Loss of PTEN is the most common mechanism of PI3K-I activation and is the second most frequently mutated tumour suppressor in human cancer (Ref. 61).

\section{Ras-MEK-ERK}

The Ras-MEK-ERK pathway is deregulated in many cancers and has a central role in mTOR activation independent of AKT. The oncogenic small GTPase Ras has an ambiguous role in autophagy. Oncogenic HRAS-V12 has been reported to suppress protein degradation via activation of PI3K-I in NIH3T3 cells (Ref. 62), whereas in nontransformed IMR90 human diploid fibroblasts, HRAS-V12 induces senescence with concomitant increased autophagy (Ref. 7). The role of ERK (MAPK1, mitogen-activated protein kinase 1) in autophagy is also multifactorial. ERK has been shown in different in vitro settings to induce autophagy (Refs 63, 64, 65). Recent findings indicate that this may occur through upregulation of Beclin 1 and destabilisation of mTOR via induction of TSC. Interestingly in this context, basal activity of MEK (MAP2K1)/ ERK conferred basal levels of mTOR and Beclin 1 that were incapable of triggering autophagy (Ref. 66). By contrast to these reports, however, it has also been shown that ERK can inhibit TSC2 and promotes tumourigenesis in vitro and in vivo (Ref. 61).

\section{STK11}

STK11 (serine/threonine kinase 11; also known as LKB1) is a tumour suppressor that is mutated in patients with hereditary intestinal polyposis
(Peutz-Jeghers syndrome), which leads to a cumulative lifetime risk of cancer at age 70 of $\sim 90 \%$ (Ref. 67). In addition, mutations in STK11 are found in a high percentage of sporadically occurring lung malignancies. STK11 is a positive regulator of autophagy through the actions of AMPK (AMP-activated protein kinase) and mTOR. Treatment of a mouse model for Peutz-Jeghers syndrome (heterozygous for Stk11) with the mTOR inhibitor rapamycin significantly reduced the tumour burden (Refs 68, 69).

\section{DAPK1}

DAPK1 (death-associated protein kinase 1) has tumour-suppressive functions and is epigenetically silenced in a variety of human tumours (Ref. 70). DAPK was believed to be a positive regulator of autophagy through interaction with LC3-interacting microtubuleassociated protein MAP1B and disruption of the Beclin-1-Bcl- $\mathrm{X}_{\mathrm{L}}$ complex (Ref. 71). Recent data, however, showed that DAPK is a positive regulator of $\mathrm{mTORC1}$ through interaction with TSC2 (Ref. 72). How this finding relates to the net effect of DAPK on autophagy has not been examined.

\section{ARHI}

The tumour suppressor ARHI (aplasia Ras homologue member I; also known as DIRAS3 for DIRAS family, GTP-binding RAS-like 3) is downregulated in more than $60 \%$ of ovarian cancer (Ref. 73). It induces autophagy by upregulation of ATG4 and blocking PI3K-I and therefore mTOR (Ref. 74). In addition, its expression leads to tumour dormancy in ovarian cancer xenograft models, which can be reversed upon treatment with chloroquine, a pharmacological inhibitor of lysosomal function and, as a result, autophagy (Ref. 74).

\section{p53}

p53 (TP53) is a major tumour suppressor, being mutated or lost in $\sim 50 \%$ of all human cancers (Ref. 75). In response to various forms of cellular stress, p53 becomes activated, leading to multiple phenotypic effects including induction of cell-cycle arrest and cell death (Ref. 76). Since p53 has been shown to respond to, and also to modulate, metabolic stress, it is perhaps no surprise that $\mathrm{p} 53$ has also been found to regulate autophagy (Refs 77, 78, 79).

Accession information: doi:10.1017/S1462399409001306; Vol. 11; e36; December 2009 (c) Cambridge University Press 2009. Re-use permitted under a Creative Commons Licence - by-nc-sa. 
The way in which p53 regulates autophagy, however, appears to be dependent on context. When elevated by cellular stress, p53 accumulates in the nucleus and activates target genes that mediate its tumour-suppressive effects. One of these genes, DRAM1 (damageregulated-autophagy modulator), encodes a phylogenetically conserved lysosomal protein that promotes autophagy (Refs 77, 80, 81). Interestingly, DRAM1 was also found to be required for p53-induced programmed cell death, although the mechanism underlying the interplay between DRAM1, autophagy and apoptosis is yet to be determined (Ref. 77). p73 (TP73), a closely related protein to p53, has also been shown to modulate DRAM1 and autophagy, although autophagy induced by p73 was found to be independent of DRAM1 (Ref. 82). Since this points to other target genes of p53/p73 being involved in autophagy regulation, it is interesting to note that sestrin 2 (SESN2) has also been linked to both p53 and p73 (Refs 83, 84). In an independent study, sestrins have been shown to modulate mTOR through AMPK (Ref. 85). A link has now also been reported between p53, sestrin 2 and autophagy (Ref. 86), although the role of sestrin 2 in p73-driven autophagy is yet to be explored.

In the absence of cellular stress, basal levels of p53 have, in contrast, been shown to inhibit autophagy. This effect, however, does not involve gene activation and occurs through a cytoplasmic mechanism at the endoplasmic reticulum (Ref. 79). Pharmacological inhibition or genetic ablation of p53, both in vitro and in vivo, was shown to induce autophagy. Although the induction and inhibition of autophagy by p53 are mechanistically distinct and therefore seemingly separable, it would be interesting to study the balance of these two effects when both are active - are they opposing or mutually exclusive? In this regard, it is interesting to note that certain tumourderived mutants of p53 can inhibit autophagy through the cytoplasmic mechanism, but they do not cause the activation of DRAM1 or sestrin 2 that would be required for the induction of autophagy (Ref. 87). In tumours with mutant p53, therefore, it would be conceivable that autophagy would be reduced through both lack of induction as well as enforced inhibition.

\section{ARF}

ARF ['alternative reading frame' of the CDKN2A (INK4A) locus] is a bona fide tumour suppressor that is known to induce p53 levels following oncogenic stress and as a result has been shown to induce p53-dependent autophagy and thereby to enhance cell death. ARF-induced autophagy might therefore contribute to its tumour-suppressive function (Ref. 88). ARF can also induce autophagy in the absence of p53, probably through interaction with $\mathrm{Bcl}-\mathrm{X}_{\mathrm{L}}$ which normally inhibits the Beclin-1-hVps34 complex (Ref. 89). A smaller truncated form of ARF, smARF (resulting from initiation from an alternative internal methionine), has also recently been found to modulate autophagy in a p53-independent manner. In this context, smARF is considered to induce autophagy at mitochondria and causes induction of a cell death dependent on autophagy genes (Ref. 90).

\section{Others}

In addition to the tumour suppressors and oncogenes discussed above, which have been shown to have an active role in autophagy regulation, several other tumour-associated factors could be postulated to modulate autophagy given their connection to pathways that have been previously implicated in autophagy regulation. As an element of speculation is involved, an exhaustive list of such factors could be generated; we feel, however, that the factors listed below are worthy of discussion even if further studies are required to clarify a connection to autophagy regulation and the extent to which this may be important for the role of the factor in tumour development.

\section{NF1}

NF1 (neurofibromin) is a tumour suppressor that antagonises Ras, and loss-of-function mutations of NF1 are associated with the familial neurofibromatosis type 1 tumour syndrome (Recklinghausen disease), which is characterised by development of benign tumours of the nervous system, as well as a more general predisposition to cancer (Ref. 61). Complicating understanding of the role of NF1 is the fact that acute loss of NF1 in human diploid fibroblasts leads to a transient upregulation of Ras, but ultimately suppresses the Ras-PI3K-I pathway and leads to

Accession information: doi:10.1017/S1462399409001306; Vol. 11; e36; December 2009 (c) Cambridge University Press 2009. Re-use permitted under a Creative Commons Licence - by-nc-sa. 
senescence. The senescent phenotype was also found in lesions from patients with neurofibromatosis. Autophagy has not been examined in this context but might well be upregulated given its crucial role in senescence (Ref. 91).

\section{REDD1}

REDD1 (regulated in development and DNAdamage responses 1; DDIT4) is induced upon hypoxia and inhibits mTORC1 through activation of TSC1/TSC2. REDD1 expression is decreased in $\sim 30 \%$ of breast and prostate carcinomas and therefore mTOR inhibition by REDD1 might prevent tumourigenesis. Indeed, xenografts with loss of REDD1 promoted tumour growth in nude mice (Refs 92, 93). Given the impact of REDD1 on mTOR (Ref. 94), it would be predicted that changes in REDD1 would affect autophagy, but this has not yet been directly addressed.

\section{Deptor}

Deptor (DEPDC6) is a newly identified mTORinteracting protein with an unknown role in autophagy. It is expressed at low levels in most cancers but high levels in multiple myeloma cells (Ref. 95). Deptor is negatively regulated by mTORC1/2. Loss of Deptor leads to activation of mTOR targets and AKT, whereas overexpression inhibits mTORC1 but activates PI3K-I-AKT signalling (Ref. 95).

\section{Summary}

In summary, the mTOR and Beclin-1-hVps34 complexes are targeted by a multitude of different oncogenes and tumour suppressors, which thereby regulate autophagy. New players are constantly being identified and new links are being made, making the regulation of autophagy in cancer incredibly complex.

\section{Mechanisms for how loss of autophagy contributes to tumourigenesis}

It is currently unclear how loss of autophagy contributes to de novo tumour formation and how to interpret autophagy in the context of already established tumours. There is, however, accumulating evidence as outlined above that autophagy is a tumour-suppressive mechanism. Several reports have now provided potential explanations as to how autophagy may function in tumour suppression, including roles in cell death, senescence and the management of metabolic stress.

\section{Autophagy and cell death}

The role and mechanisms of autophagy in promoting cell survival in contexts such as nutrient deprivation are unequivocal. It is also without question that autophagy accompanies cell death in certain scenarios, although the exact contribution of autophagy is complex and less well defined. In systems where apoptosis was inhibited, either genetically or pharmacologically, cell death dependent on ATGs was reported (Refs 96, 97); in other words, inhibition of certain ATGs (ATG5, Beclin 1, ATG7) suppressed cell death. In one report, where cells were treated with the caspase inhibitor zVAD-fmk to inhibit caspasedependent death, it was proposed that the cell death was mechanistically driven via the selective autophagic degradation of catalase, which subsequently overloads the cell with damaging ROS (Ref. 98). A more direct role of ATGs in cell death has also been shown: cleavage of ATG5 has been reported to occur following cell death induction and this cleaved form has a direct proapoptotic role (Ref. 99). This could indicate, therefore, that in studies where cell death has been compromised by ATG5 knockdown, incorrect conclusions may have been drawn that the cell death observed was dependent on autophagy. The generality of these observations, however, is yet to be determined.

Clearance of dying cells during salivary gland development in Drosophila melanogaster involves autophagy and in this context it appears that the full-blown cell death response is dependent on caspases and ATGs (Ref. 100). Increasing evidence also suggests a non-cell-autonomous role of autophagy in cell death, which extends the housekeeping functions of autophagy to a multicellular level. In an in vitro mouse model of embryonic development, inhibition of autophagy prevented the upregulation of two important cellsurface engulfment signals - phosphatidylserine and lysophosphatidylcholine - in cells destined to die (Ref. 101). At first impression, inhibition of autophagy in this system seems to result in cell death, but it is really the energy-dependent expression of engulfment signals that is impeded, which causes the accumulation of cellular corpses by default, that is at play. Exogenous methylpyruvate is an alternative

Accession information: doi:10.1017/S1462399409001306; Vol. 11; e36; December 2009 (c) Cambridge University Press 2009. Re-use permitted under a Creative Commons Licence - by-nc-sa. 
source of energy and its addition compensates for the low levels of ATP in autophagy-deficient cells, leading to expression of the aforementioned engulfment signals. In an vivo context, it is notable that embryonic Atg5-null mice display reduced phagocytosis of dead cells in lung and retinal tissue, pointing to the wider implications of this phenomenon and to the caution that should be employed when dying cells seemingly arise following autophagy inhibition (Ref. 101).

In all the above-mentioned examples, ATGs facilitate cell death, but it is arguable that a situation has not yet been observed where a cell actually dies solely from autophagy. Furthermore, as discussed above, the role of autophagy in cell death is not restricted to cell-autonomous effects. Therefore, the term 'autophagic cell death' (or programmed cell death type II) has to be used with caution. It has been suggested it be replaced with the term 'cell death with autophagy' as it is now widely considered that autophagy can be a component in cell death, perhaps as a permissive signal, but not its sole perpetrator (Ref. 102).

\section{Autophagy in cell cycle arrest, dormancy and senescence}

Sustained growth arrest, like programmed cell death, is an important mechanism to counteract tumour growth in vivo and has recently been shown to be critically linked with autophagy (Ref. 100). Senescence is a critical barrier against malignant transformation and an important effector programme of chemotherapy. It is defined as an irreversible cell cycle arrest of viable and metabolically active cells following various endogenous and exogenous stresses (Ref. 103). In HRAS-V12-infected human diploid fibroblasts, autophagy is activated and its inhibition resulted in delayed onset of the senescent phenotype (Ref. 7). In a reciprocal fashion, overexpression of the autophagic protein ULK3 induced autophagy and accelerated the onset of senescence (Ref. 7). However, the exact role of ULK3 in autophagy is currently unclear.

Tumour dormancy generally describes a phase of apparent stasis in tumour growth and is mechanistically subdivided into two models. Single-cell dormancy is a phase of prolonged cell cycle arrest that can be overcome upon cellautonomous genetic changes or alterations in the microenvironment. According to the micrometastasis model, tumour dormancy is a balanced state of cell renewal and cell death (Ref. 104). (Both dormancy and senescence are not to be confused with quiescence, which is a physiological normal resting state of a cell.) ARHI expression induces autophagy and leads to tumour dormancy in ovarian cancer xenograft models, which can be reversed upon treatment with chloroquine, a pharmacological inhibitor of lysosomal function and therefore autophagy (Ref. 74).

When taken together, these reports clearly emphasise that growth arrest and autophagy are intertwined, and tentatively posit that autophagy and its role in senescence and dormancy may be a facet of tumour suppression in multiple cancers.

\section{Autophagy and the management of oxidative stress}

Metabolic stress, as a result of either insufficient nutrient/oxygen supply or increased energetic demands of rapidly dividing tumour cells, induces autophagy as an alternative source of energy and metabolites. Enhanced autophagy is frequently found in hypoxic regions of tumours and contributes to cell survival (Ref. 105). Initially, it is therefore illogical how loss of autophagy contributes to tumour formation (Refs 32, 34, 35). The first contribution to solve this conundrum came from the observation that metabolic stress promotes necrotic cell death in vivo and in vitro within cells that are defective in both autophagy and apoptosis. Necrotic cell death was associated with a strong inflammatory response, which ultimately enhanced tumour growth (Ref. 105). This therefore provides one explanation of how autophagy may be tumour suppressive through its capacity to impede necrosis. Whether this is a factor, however, downstream of chemotherapeutic drugs that induce cell death through necrosis is an interesting question and worthy of further investigation (Refs 106, 107). Meanwhile it is becoming increasingly substantiated that autophagy reduces oxidative stress, maintains protein and organelle quality control and thereby limits cellular damage (Refs 27, 28).

Metabolic stress leads to accumulation of ROS, damaged proteins and damaged organelles such as mitochondria (which are in turn additional

Accession information: doi:10.1017/S1462399409001306; Vol. 11; e36; December 2009 (c) Cambridge University Press 2009. Re-use permitted under a Creative Commons Licence - by-nc-sa. 
sources of ROS) and impedes genetic stability when compensatory mechanisms fail. Autophagy is a critical countermeasure following metabolic insults and attenuates these changes, thereby promoting cellular survival. In different studies, autophagy-defective tumour cells were indeed more susceptible to cell death but, importantly, at the same time were also more susceptible to genomic damage and possessed greatly enhanced tumourigenic potential when compared with autophagycompetent cells in vivo (Refs 27, 28).

Sequestosome 1 (SQSTM1; p62) has been identified as the critical molecular link between defective autophagy, genomic instability and tumourigenesis (Ref. 26). p62 is an adapter protein that links polyubiquitinated proteins or aggregates to ATG8/LC3 on the surface of autophagosomes. Importantly, it is also a decisive element in modulating different molecular pathways, such as the nuclear factor NF-кB pathway through recruitment of different signalling molecules and the extrinsic cell death pathway by promoting aggregation of caspase 8 (Ref. 108). p62 is frequently upregulated in human tumours and of utmost importance in controlling oxidative stress and tumour growth (Refs 26, 109). Tumour cells, therefore, with impairment in both apoptosis and autophagy preferably accumulate p62 under stress, which thereby promotes tumourigenesis (Ref. 26). In a self-supporting feedback loop, metabolic stress leads to increased production of ROS that in turn were responsible for accumulation of p62. p62 itself was also causative for further oxidative damage, accumulation of damaged mitochondria, an enhanced induction of the protein-folding machinery in the endoplasmatic reticulum, and a DNA-damage response. Elevated levels of p62 suppressed the activation of target genes of NF-кB. In Ras-induced lung tumours, p62 is essential for tumour formation, but in contrast to the observation by Mathew et al. (Ref. 26), inhibition of p62 led to impaired NF- $\kappa$ B signalling (Ref. 110). These differences in NF- $\mathrm{B}$ activation are not readily explained and may be due to the versatility of p62 in activating different pathways (Ref. 109) or the different cellular contexts. Nonetheless, p62 accumulation is clearly causative for tumourigenesis and exemplifies how cancer progression can be affected by the selective autophagic degradation of a specific protein. Figure 4 illustrates how the metabolic capacity of autophagy might be the decisive factor for proliferation control in tumourigenesis.

\section{Autophagy and therapy - whether to treat and, if so, how?}

With regard to the complexity and dichotomy of autophagy in cell death and tumour biology and limited treatment data from in vivo studies, can autophagy be modulated for the benefit of cancer therapy? As a result of the survival effects of autophagy that were initially observed in vitro, the suggestion would have been that autophagy inhibition, in combination with standard chemotherapy, would be beneficial for tumour therapy. When we consider, however, the effects this may have on a necrosis-driven protumour inflammatory response or the effects on impeding cellular senescence, one would perhaps aver that autophagy promotion would be the way to go. Nonetheless, several recent reports have shown that pharmacological inhibition of autophagy with chloroquine might be beneficial for tumour therapy (Refs 74, 111). The effects of chloroquine are, however, not limited to autophagy inhibition, causing, among other things, inhibition of lysosomal function in general, and it is important to note that in vivo genetic verification that the effects of chloroquine in these settings was through modulation of autophagy is yet to be established. In this regard, it is notable that a separate study analysing the therapeutic effectiveness of chloroquine revealed prodeath effects of autophagy. In this context, inhibition of caspases in an autophagy-deficient background, but not in autophagy-competent cells, reduced the cell death seen following chloroquine treatment. This would indicate, therefore, that cell death downstream of chloroquine in this setting is, in part, autophagy dependent (Ref. 112).

Another study has also recently examined the regulation of autophagy by a doxycycline (Dox)-regulatable ARHI-encoding gene (DIRAS3) in xenografts. Treatment of mice carrying these xenografts with Dox causes induction of ARHI and autophagy and this led to reduced tumour growth compared with untreated animals. Upon removal of Dox, ARHI and autophagy were reduced and rapid tumour outgrowth was observed. However, when ARHI

Accession information: doi:10.1017/S1462399409001306; Vol. 11; e36; December 2009 (c) Cambridge University Press 2009. Re-use permitted under a Creative Commons Licence - by-nc-sa. 


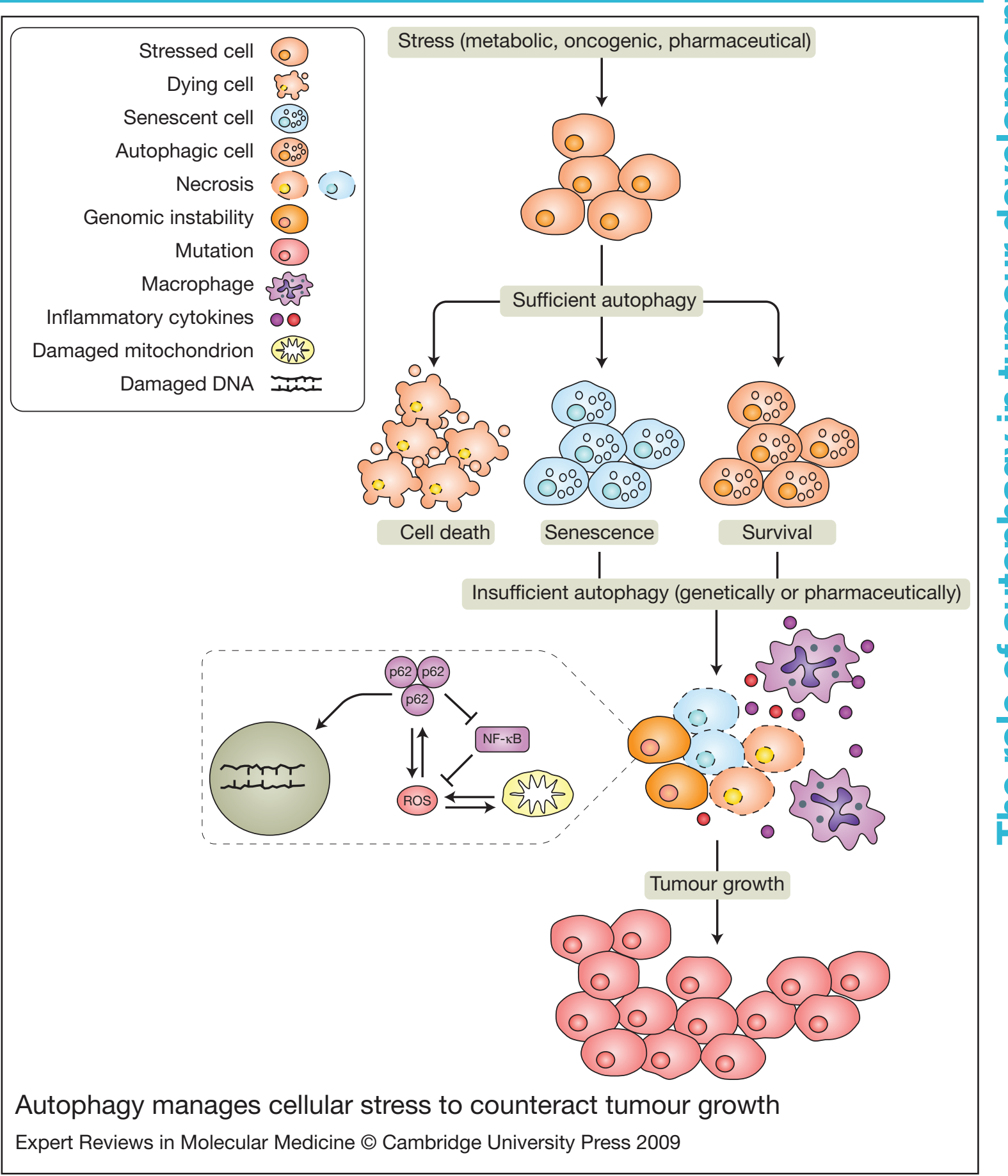

Figure 4. Autophagy manages cellular stress to counteract tumour growth. In autophagy-competent cells, cellular stress leads either to cell death, growth arrest in the form of premature senescence, or survival. If the autophagic capacity falls below a threshold that is necessary to maintain cell integrity, for example through genetic alterations or pharmaceutical intervention, cells are unable to compensate metabolic stress. As a result, necrotic cell death occurs and causes attraction of tumour-promoting macrophages. In surviving cells, however, failure to clear p62 (sequestosome 1) and p62-associated aggregates results in further accumulation of ROS (reactive oxygen species), damaged proteins and organelles, altered cell signalling and DNA damage. Clinically, this phase might present as an initial tumour remission. However, the acquisition of growth-promoting mutations in a subset of cells might potentially lead to more aggressive tumour cells. Abbreviation: NF-кB, nuclear factor $\kappa B$.

Accession information: doi:10.1017/S1462399409001306; Vol. 11; e36; December 2009 (c) Cambridge University Press 2009. Re-use permitted under a Creative Commons Licence - by-nc-sa. 
expression was induced in mice treated with chloroquine at the same time to inhibit autophagy, xenograft growth was rapidly diminished after withdrawal of Dox (and chloroquine) (Ref. 74). Taken together, a possible interpretation of these finding is that 'genetic' and pharmacological ablation of autophagy produce different results and again point towards alternative or additional therapeutic effects of chloroquine in different contexts. More studies are therefore required to understand the relevance of autophagy following treatment with chloroquine in different settings.

When considering targeting autophagy therapeutically, the effects on normal as well as tumour cells must always be considered. This is no more relevant than with autophagy, which has major homeostatic roles within our normal tissues. For example, the selective ablation of autophagy in the brains of mice has been shown to lead to neurodegenerative disease without any other accompanying mutation, indicating that the systemic modulation of autophagy may be naive (Refs 37,113 ). As a result, it seems clear that we perhaps need to understand more about the selective control of autophagy in different settings to enable the bespoke targeting of autophagy to hit diseased cells, but not normal tissue. An understanding of the signalling pathways that talk to the autophagy machinery in response to different forms of cellular stress to bring about different effects may therefore be the key. In this regard, a recent report has identified that signalling from PDGF (platelet-derived growth factor) receptors is a permissive signal for hypoxiadriven autophagy while seemingly having no effects on autophagy driven by other stimuli. Since autocrine PDGFR signalling and hypoxia are both tumour-associated events this may well be a paradigm for the identification of autophagic signalling pathways that could be utilised for targeting tumour-selective autophagy, and further studies of this, and other pathways, undoubtedly merit further investigation (Refs 114, 115; see also Fig. 2).

In summary, autophagy is important for development and cellular homeostasis, and lies at the intersection of life and death. More and more evidence is accumulating, however, that autophagy is a tumour-suppressive mechanism, with bonds to the control of oxidative damage, cell death and oncogene-induced senescence. Looking at autophagy from a metabolism angle provides explanation of how loss of a cytoprotective mechanism facilitates tumour development through mitigating genomic stress. In line with these desired tumour-suppressive functions, autophagy-enhancing therapy may be as promising a therapeutic strategy as the simple notion of inhibiting autophagy to enhance cell death. It is undoubted, however, that our current understanding of autophagy is incomplete. As more genetic models of autophagy inhibition are developed, which allow the spatial and temporal control of autophagy, this should enable the more effective targeting of autophagy in a both a selective and bespoke manner.

\section{Acknowledgements and funding}

We are grateful to members of the Tumour Cell Death Laboratory for critical reading of the manuscript. We also thank the peer reviewers of our article, whose insightful comments improved the clarity and comprehensive nature of this review. Work in the Tumour Cell Death Laboratory is supported by Cancer Research UK and the Association for International Cancer Research.

\section{Note added in proof}

The reader should be aware that in the context of autophagy regulation as described in this review, and despite what was previously believed, a recent report has indicated that autophagy can occur in the absence of both ATG5 and ATG7 (Ref. 116). The generality of this observation and what it means in terms of the genetic analysis of autophagy as a result of depletion/loss of ATG5, ATG7 or both are certainly areas that merit further investigation.

\section{References}

1 Lum, J.J. et al. (2005) Growth factor regulation of autophagy and cell survival in the absence of apoptosis. Cell 120, 237-248

2 Lum, J.J., DeBerardinis, R.J. and Thompson, C.B. (2005) Autophagy in metazoans: cell survival in the land of plenty. Nature Reviews Molecular Cell Biology 6, 439-448

3 Kuma, A. et al. (2004) The role of autophagy during the early neonatal starvation period. Nature 432 , 1032-1036

Accession information: doi:10.1017/S1462399409001306; Vol. 11; e36; December 2009 (c) Cambridge University Press 2009. Re-use permitted under a Creative Commons Licence - by-nc-sa. 
4 Mizushima, N. et al. (2008) Autophagy fights disease through cellular self-digestion. Nature 451, 1069-1075

5 Maiuri, M.C. et al. (2009) Control of autophagy by oncogenes and tumor suppressor genes. Cell Death and Differentiation 16, 87-93

6 Maiuri, M.C. et al. (2007) Self-eating and self-killing: crosstalk between autophagy and apoptosis. Nature Reviews Molecular Cell Biology $8,741-752$

7 Young, A.R.J. et al. (2009) Autophagy mediates the mitotic senescence transition. Genes and Development 23, 798-803

8 Kondo, Y. et al. (2005) The role of autophagy in cancer development and response to therapy. Nature Reviews Cancer 5, 726-734

9 Eskelinen, E-L. (2005) Maturation of autophagic vacuoles in Mammalian cells. Autophagy 1, 1-10

10 Xie, Z. and Klionsky, D.J. (2007) Autophagosome formation: core machinery and adaptations. Nature Cell Biology 9, 1102-1109

11 Levine, B. and Kroemer, G. (2008) Autophagy in the pathogenesis of disease. Cell 132, 27-42

12 Diaz-Troya, S. et al. (2008) The role of TOR in autophagy regulation from yeast to plants and mammals. Autophagy 4, 851-865

13 Hara, T. et al. (2008) FIP200, a ULK-interacting protein, is required for autophagosome formation in mammalian cells. Journal of Cell Biology 181, 497-510

14 Jung, C.H. et al. (2009) ULK-Atg13-FIP200 complexes mediate mTOR signaling to the autophagy machinery. Molecular Biology of the Cell 20, 1992-2003

15 Ganley, I.G. et al. (2009) ULK1.ATG13.FIP200 complex mediates mTOR signaling and is essential for autophagy. Journal of Biological Chemistry 284, 12297-12305

16 Liang, C. et al. (2006) Autophagic and tumour suppressor activity of a novel Beclin1-binding protein UVRAG. Nature Cell Biology 8, 688-699

17 Liang, C. et al. (2008) Beclin1-binding UVRAG targets the class C Vps complex to coordinate autophagosome maturation and endocytic trafficking. Nature Cell Biology 10, 776-787

18 Ohsumi, Y. and Mizushima, N. (2004) Two ubiquitin-like conjugation systems essential for autophagy. Seminars in Cell and Developmental Biology 15, 231-236

19 Geng, J. and Klionsky, D.J. (2008) The Atg8 and Atg12 ubiquitin-like conjugation systems in macroautophagy. 'Protein modifications: beyond the usual suspects' review series. EMBO Reports 9, 859-864

20 Kabeya, Y. et al. (2000) LC3, a mammalian homologue of yeast Apg8p, is localized in autophagosome membranes after processing. EMBO Journal 19, 5720-5728

21 Klionsky, D.J. et al. (2008) Guidelines for the use and interpretation of assays for monitoring autophagy in higher eukaryotes. Autophagy 4, 151-175

22 Xie, Z., Nair, U. and Klionsky, D.J. (2008) Atg8 controls phagophore expansion during autophagosome formation. Molecular Biology of the Cell 19, 3290-3298

23 Yang, Z. et al. (2006) Atg22 recycles amino acids to link the degradative and recycling functions of autophagy. Molecular Biology of the Cell 17, 5094-5104

24 Hanahan, D. and Weinberg, R.A. (2000) The hallmarks of cancer. Cell 100, 57-70

25 Mathew, R., Karantza-Wadsworth, V. and White, E. (2007) Role of autophagy in cancer. Nature Reviews Cancer 7, 961-967

26 Mathew, R. et al. (2009) Autophagy suppresses tumorigenesis through elimination of p62. Cell 137, 1062-1075

27 Karantza-Wadsworth, V. et al. (2007) Autophagy mitigates metabolic stress and genome damage in mammary tumorigenesis. Genes and Development 21, 1621-1635

28 Mathew, R. et al. (2007) Autophagy suppresses tumor progression by limiting chromosomal instability. Genes and Development 21, 1367-1381

29 Jin, S. and White, E. (2007) Role of autophagy in cancer: management of metabolic stress.

Autophagy 3, 28-31

30 Eisenberg-Lerner, A. and Kimchi, A. (2009) The paradox of autophagy and its implication in cancer etiology and therapy. Apoptosis 14, 376-391

31 Aita, V.M. et al. (1999) Cloning and genomic organization of beclin 1, a candidate tumor suppressor gene on chromosome 17q21. Genomics 59, 59-65

32 Liang, X.H. et al. (1999) Induction of autophagy and inhibition of tumorigenesis by beclin 1 . Nature $402,672-676$

33 Miracco, C. et al. (2007) Protein and mRNA expression of autophagy gene Beclin 1 in human brain tumours. International Journal of Oncology 30, 429-436

$34 \mathrm{Qu}, \mathrm{X}$. et al. (2003) Promotion of tumorigenesis by heterozygous disruption of the beclin 1

Accession information: doi:10.1017/S1462399409001306; Vol. 11; e36; December 2009 
autophagy gene. Journal of Clinical

Investigation 112, 1809-1820

35 Yue, Z. et al. (2003) Beclin 1, an autophagy gene essential for early embryonic development, is a haploinsufficient tumor suppressor. Proceedings of the National Academy of Sciences of the United States of America 100, 15077-15082

36 Komatsu, M. et al. (2005) Impairment of starvationinduced and constitutive autophagy in Atg7-deficient mice. Journal of Cell Biology 169, 425-434

37 Hara, T. et al. (2006) Suppression of basal autophagy in neural cells causes neurodegenerative disease in mice. Nature 441, 885-889

38 Mariño, G. et al. (2007) Tissue-specific autophagy alterations and increased tumorigenesis in mice deficient in Atg4C/autophagin-3. Journal of Biological Chemistry 282, 18573-18583

39 Kim, M.S. et al. (2008) Frameshift mutation of UVRAG, an autophagy-related gene, in gastric carcinomas with microsatellite instability. Human Pathology 39, 1059-1063

40 Kang, M.R. et al. (2009) Frameshift mutations of autophagy-related genes ATG2B, ATG5, ATG9B and ATG12 in gastric and colorectal cancers with microsatellite instability. Journal of Pathology 217, 702-706

41 Takahashi, Y. et al. (2007) Bif-1 interacts with Beclin 1 through UVRAG and regulates autophagy and tumorigenesis. Nature Cell Biology 9, 1142-1151

42 Morselli, E. et al. (2009) Anti- and pro-tumor functions of autophagy. Biochimica et Biophysica Acta 1793, 1524-1532

43 Pattingre, S. et al. (2008) Regulation of macroautophagy by mTOR and Beclin 1 complexes. Biochimie 90, 313-323

44 Vogt, P.K., Gymnopoulos, M. and Hart, J.R. (2009) PI 3-kinase and cancer: changing accents. Current Opinion in Genetics and Development $19,12-17$

45 Martin, D.E. and Hall, M.N. (2005) The expanding TOR signaling network. Current Opinion in Cell Biology 17, 158-166

$46 \mathrm{Kim}$, D.H. et al. (2002) mTOR interacts with raptor to form a nutrient-sensitive complex that signals to the cell growth machinery. Cell 110, 163-175

47 Sarbassov, D.D. et al. (2004) Rictor, a novel binding partner of $\mathrm{mTOR}$, defines a rapamycininsensitive and raptor-independent pathway that regulates the cytoskeleton. Current Biology $14,1296-1302$

48 Sarbassov, D.D. et al. (2005) Phosphorylation and regulation of $\mathrm{Akt} / \mathrm{PKB}$ by the rictor-mTOR complex. Science 307, 1098-1101
49 Toschi, A. et al. (2008) Differential dependence of hypoxia-inducible factors 1 alpha and 2 alpha on mTORC1 and mTORC2. Journal of Biological Chemistry 283, 34495-34499

50 Guertin, D.A. and Sabatini, D.M. (2007) Defining the role of mTOR in cancer. Cancer Cell 12, 9-22

51 Levine, B., Sinha, S. and Kroemer, G. (2008) Bcl-2 family members: dual regulators of apoptosis and autophagy. Autophagy 4, 600-606

52 Maiuri, M.C. et al. (2007) Functional and physical interaction between $\mathrm{Bcl}-\mathrm{X}(\mathrm{L})$ and a $\mathrm{BH} 3-$

like domain in Beclin-1. EMBO Journal 26, 2527-2539

53 Pattingre, S. et al. (2005) Bcl-2 antiapoptotic proteins inhibit Beclin 1-dependent autophagy. Cell 122, 927-939

54 Itakura, E. et al. (2008) Beclin 1 forms two distinct phosphatidylinositol 3-kinase complexes with mammalian Atg14 and UVRAG. Molecular Biology of the Cell 19, 5360-5372

55 Sun, Q. et al. (2008) Identification of Barkor as a mammalian autophagy-specific factor for Beclin 1 and class III phosphatidylinositol 3-kinase. Proceedings of the National Academy of Sciences of the United States of America 105, 19211-19216

56 Zhong, Y. et al. (2009) Distinct regulation of autophagic activity by Atg14L and Rubicon associated with Beclin 1-phosphatidylinositol-3kinase complex. Nature Cell Biology 11, 468-476

57 Matsunaga, K. et al. (2009) Two Beclin 1-binding proteins, Atg14L and Rubicon, reciprocally regulate autophagy at different stages. Nature Cell Biology 11, 385-396

58 Fimia, G.M. et al. (2007) Ambra1 regulates autophagy and development of the nervous system. Nature 447, 1121-1125

59 Yuan, T.L. and Cantley, L.C. (2008) PI3K pathway alterations in cancer: variations on a theme. Oncogene 27, 5497-5510

60 Krymskaya, V.P. and Goncharova, E.A. (2009) $\mathrm{PI} \mathrm{K} / \mathrm{mTORC} 1$ activation in hamartoma syndromes: therapeutic prospects. Cell Cycle 8, 403-413

61 Shaw, R.J. and Cantley, L.C. (2006) Ras, PI(3)K and mTOR signalling controls tumour cell growth. Nature 441, 424-430

62 Furuta, S. et al. (2004) Ras is involved in the negative control of autophagy through the class I PI3-kinase. Oncogene 23, 3898-3904

63 Ogier-Denis, E. et al. (2000) Erk1/2-dependent phosphorylation of Galpha-interacting protein stimulates its GTPase accelerating 
activity and autophagy in human colon cancer cells. Journal of Biological Chemistry 275, 39090-39095

64 Pattingre, S., Bauvy, C. and Codogno, P. (2003) Amino acids interfere with the ERK1/2-dependent control of macroautophagy by controlling the activation of Raf-1 in human colon cancer HT-29 cells. Journal of Biological Chemistry 278, 16667-16674

65 Corcelle, E. et al. (2006) Disruption of autophagy at the maturation step by the carcinogen lindane is associated with the sustained mitogen-activated protein kinase/extracellular signal-regulated kinase activity. Cancer Research 66, 6861-6870

66 Wang, J. et al. (2009) A non-canonical MEK/ERK signaling pathway regulates autophagy via regulating Beclin 1. Journal of Biological Chemistry 284, 21412-21424

67 Rustgi, A.K. (2007) The genetics of hereditary colon cancer. Genes and Development 21, 2525-2538

68 Liang,J.etal.(2007) The energy sensing LKB1-AMPK pathway regulates p27(kip1) phosphorylation mediating the decision to enter autophagy or apoptosis. Nature Cell Biology 9, 218-224

69 Wei, C. et al. (2008) Suppression of Peutz-Jeghers polyposis by targeting mammalian target of rapamycin signaling. Clinical Cancer Research 14, 1167-1171

70 Gozuacik, D. and Kimchi, A. (2006) DAPk protein family and cancer. Autophagy 2, 74-79

71 Zalckvar, E. et al. (2009) DAP-kinase-mediated phosphorylation on the $\mathrm{BH} 3$ domain of beclin 1 promotes dissociation of beclin 1 from Bcl-XL and induction of autophagy. EMBO Reports 10, 285-292

72 Stevens, C. et al. (2009) Peptide combinatorial libraries identify TSC2 as a death-associated protein kinase (DAPK) death domain-binding protein and reveal a stimulatory role for DAPK in mTORC1 signaling. Journal of Biological Chemistry 284, 334-344

73 Yu, Y. et al. (2003) Epigenetic regulation of ARHI in breast and ovarian cancer cells. Annals of the New York Academy of Sciences 983, 268-277

$74 \mathrm{Lu}, \mathrm{Z}$. et al. (2008) The tumor suppressor gene ARHI regulates autophagy and tumor dormancy in human ovarian cancer cells. Journal of Clinical Investigation 118, 3917-3929

75 Hainaut, P. and Hollstein, M. (2000) p53 and human cancer: the first ten thousand mutations. Advances in Cancer Research 77, 81-137
76 Crighton, D. and Ryan, K.M. (2004) Splicing DNAdamage responses to tumour cell death. Biochimica et Biophysica Acta 1705, 3-15

77 Crighton, D. et al. (2006) DRAM, a p53-induced modulator of autophagy, is critical for apoptosis. Cell 126, 121-134

78 Feng, Z. et al. (2005) The coordinate regulation of the p53 and mTOR pathways in cells. Proceedings of the National Academy of Sciences of the United States of America 102, 8204-8209

79 Tasdemir, E. et al. (2008) Regulation of autophagy by cytoplasmic p53. Nature Cell Biology 10, 676-687

80 Crighton, D., Wilkinson, S. and Ryan, K.M. (2007) DRAM links autophagy to p53 and programmed cell death. Autophagy 3, 72-74

81 O'Prey, J. et al. (2009) Analysis of DRAM-related proteins reveals evolutionarily conserved and divergent roles in the control of autophagy. Cell Cycle 8, 2260-2265

82 Crighton, D. et al. (2007) p73 regulates DRAMindependent autophagy that does not contribute to programmed cell death. Cell Death and Differentiation 14, 1071-1079

83 Budanov, A.V. et al. (2004) Regeneration of peroxiredoxins by p53-regulated sestrins, homologs of bacterial AhpD. Science 304, 596-600

84 Rosenbluth, J.M. et al. (2008) A gene signaturebased approach identifies $\mathrm{mTOR}$ as a regulator of p73. Molecular and Cellular Biology 28, 5951-5964

85 Budanov, A.V. and Karin, M. (2008) p53 target genes sestrin 1 and sestrin2 connect genotoxic stress and mTOR signaling. Cell 134, 451-460

86 Maiuri, M.C. et al. (2009) Stimulation of autophagy by the p53 target gene Sestrin2. Cell Cycle 8, 1571-1576

87 Morselli, E. et al. (2008) Mutant p53 protein localized in the cytoplasm inhibits autophagy. Cell Cycle 7, 3056-3061

88 Abida, W.M. and Gu, W. (2008) p53-Dependent and p53-independent activation of autophagy by ARF. Cancer Research 68, 352-357

89 Pimkina, J. et al. (2009) ARF induces autophagy by virtue of interaction with Bcl-xl. Journal of Biological Chemistry 284, 2803-2810

90 Reef, S. et al. (2006) A short mitochondrial form of p19ARF induces autophagy and caspase-independent cell death. Molecular Cell $22,463-475$

91 Courtois-Cox, S. et al. (2006) A negative feedback signaling network underlies oncogeneinduced senescence. Cancer Cell 10, 459-472 
92 DeYoung, M.P. et al. (2008) Hypoxia regulates TSC1/2-mTOR signaling and tumor suppression through REDD1-mediated 14-3-3 shuttling. Genes and Development 22, 239-251

93 Memmott, R.M. and Dennis, P.A. (2009) Aktdependent and -independent mechanisms of $\mathrm{mTOR}$ regulation in cancer. Cellular Signalling 21, 656-664

94 Brugarolas, J. et al. (2004) Regulation of mTOR function in response to hypoxia by REDD1 and the TSC1/TSC2 tumor suppressor complex. Genes and Development 18, 2893-2904

95 Peterson, T.R. et al. (2009) DEPTOR is an mTOR inhibitor frequently overexpressed in multiple myeloma cells and required for their survival. Cell $137,873-886$

96 Shimizu, S. et al. (2004) Role of Bcl-2 family proteins in a non-apoptotic programmed cell death dependent on autophagy genes. Nature Cell Biology 6, 1221-1228

$97 \mathrm{Yu}$, L. et al. (2004) Regulation of an ATG7-beclin 1 program of autophagic cell death by caspase-8. Science 304, 1500-1502

$98 \mathrm{Yu}$, L. et al. (2006) Autophagic programmed cell death by selective catalase degradation. Proceedings of the National Academy of Sciences of the United States of America 103, 4952-4957

99 Yousefi, S. et al. (2006) Calpain-mediated cleavage of Atg5 switches autophagy to apoptosis. Nature Cell Biology 8, 1124-1132

100 Berry, D.L. and Baehrecke, E.H. (2007) Growth arrest and autophagy are required for salivary gland cell degradation in Drosophila. Cell 131, $1137-1148$

$101 \mathrm{Qu}$, X. et al. (2007) Autophagy gene-dependent clearance of apoptotic cells during embryonic development. Cell 128, 931-946

102 Kroemer, G. and Levine, B. (2008) Autophagic cell death: the story of a misnomer. Nature Reviews Molecular Cell Biology 9, 1004-1010

103 Prieur, A. and Peeper, D.S. (2008) Cellular senescence in vivo: a barrier to tumorigenesis. Current Opinion in Cell Biology 20, 150-155

104 Fehm, T. et al. (2008) Tumor cell dormancy: implications for the biology and treatment of breast cancer. APMIS: Acta Pathologica Microbiologica et Immunologica Scandinavica 116, 742-753
105 Degenhardt, K. et al. (2006) Autophagy promotes tumor cell survival and restricts necrosis, inflammation, and tumorigenesis. Cancer Cell 10, 51-64

106 Amaravadi, R.K. and Thompson, C.B. (2007) The roles of therapy-induced autophagy and necrosis in cancer treatment. Clinical Cancer Research 13, 7271-7279

107 Zong, W.X. et al. (2004) Alkylating DNA damage stimulates a regulated form of necrotic cell death. Genes and Development 18, 1272-1282

108 Jin, Z. et al. (2009) Cullin3-based polyubiquitination and p62-dependent aggregation of caspase- 8 mediate extrinsic apoptosis signaling. Cell 137, 721-735

109 Moscat, J. and Diaz-Meco, M.T. (2009) p62 at the crossroads of autophagy, apoptosis, and cancer. Cell 137, 1001-1004

110 Duran, A. et al. (2008) The signaling adaptor p62 is an important NF-kappaB mediator in tumorigenesis. Cancer Cell 13, 343-354

111 Amaravadi, R.K. et al. (2007) Autophagy inhibition enhances therapy-induced apoptosis in a Myc-induced model of lymphoma. Journal of Clinical Investigation 117, 326-336

112 Maclean, K.H. et al. (2008) Targeting lysosomal degradation induces $\mathrm{p} 53$-dependent cell death and prevents cancer in mouse models of lymphomagenesis. Journal of Clinical Investigation 118, 79-88

113 Komatsu, M. et al. (2006) Loss of autophagy in the central nervous system causes neurodegeneration in mice. Nature 441, 880-884

114 Wilkinson, S. et al. (2009) Hypoxia-selective macroautophagy and cell survival signaled by autocrine PDGFR activity. Genes and Development 23, 1283-1288

115 Wilkinson, S. and Ryan, K.M. (2009) Growth factor signaling permits hypoxia-induced autophagy by a HIF1alpha-dependent, BNIP3/3L-independent transcriptional program in human cancer cells. Autophagy 5, 1068-1069

116 Nishida, Y. et al. (2009) Discovery of Atg5/Atg7independent alternative macroautophagy. Nature 461, 654-659 


\section{Further reading, resources and contacts}

A newly established forum on autophagy, hosted by the journal Autophagy, provides useful information on reagents and antibodies used in autophagy research:

http://www.landesbioscience.com/journals/autophagy/forum/

The US National Cancer Institute website might prove useful for clinicians to gain a rapid overview of research and clinical trials that are ongoing in the autophagy field in the USA:

http://www.cancer.gov/

Likewise, the Cancer Research UK website provides similar information about research projects on autophagy in the UK:

http://www.cancerresearchuk.org/

Further information about work in the authors' laboratory and about The Beatson Institute for Cancer Research in general can be found at:

http://www.beatson.gla.ac.uk/

\section{Features associated with this article}

\section{Figures}

Figure 1. Mechanisms of autophagy.

Figure 2. Signalling networks of oncogenes and tumour suppressors that control autophagy.

Figure 3. Regulation of autophagy by Beclin 1 complexes.

Figure 4. Autophagy manages cellular stress to counteract tumour growth.

\section{Citation details for this article}

Mathias T. Rosenfeldt and Kevin M. Ryan (2009) The role of autophagy in tumour development and cancer therapy. Expert Rev. Mol. Med. Vol. 11, e36, December 2009, doi:10.1017/S1462399409001306 